\title{
STABILITY OF RICCI FLOWS BASED ON KILLING CONDITIONS
}

\author{
Peibiao Zhao and Qihui Cai
}

\begin{abstract}
C. Guenther studied the stability of DeTurck flows by using maximal regularity theory and center manifolds, but these arguments can not solve the stability of Ricci flows because the Ricci flow equation is not strictly parabolic. Recognizing this deficiency, the present paper considers and obtains the stability of Ricci flows, and of quasi-Ricci flows in view of some Killing conditions.
\end{abstract}

\section{Introduction}

The study of Ricci flows has been an active field over the past several decades. R. Hamilton [12], drawing inspiration from the work by Elles and Sampson [9], introduced the well-known Ricci flows as follows:

$$
\frac{\partial}{\partial t} g=-2 R c[g], g(0)=g_{0} .
$$

R. Hamilton posed that if a closed Riemannian manifold with dimension 3 has an initial metric $g_{0}$ with positive Ricci curvature, then the solution $g(t)$, for all time $t$, of normalized Ricci flow exists and $g(t)$ converges exponentially fast to a constant scalar curvature metric $g_{\infty}$. In this setting, it is not hard to see that for a given arbitrary homotopy sphere with dimension 3 , if it has a metric with positive Ricci curvature, then Poincaré conjecture is tenable.

As we know that it is a fundamental and difficult problem in differential geometry to find a standard metric satisfying some prescribed conditions over a Riemannian manifold. For instance, concerning the celebrated Yamabe problem [20], it is essential to find a metric with constant scalar curvature; For constant Ricci curvature, one needs to solve Einstein equation. The study of Ricci flows, in general, is exactly to find a standard metric satisfying the given

Received February 18, 2008; Revised May 22, 2008.

2000 Mathematics Subject Classification. 28A22, 53C12.

Key words and phrases. Ricci flows, quasi-Ricci flows, DeTurck flows, Killing conditions. Supported by a Grant-in-Aid for Scientific Research from Nanjing University of Science and Technology (XKF09042, KN11008) and by NNSF(10771102).

Supported by a Grant-in-Aid for Scientific Research from Nanjing University of Science and Technology (AB41409). 
conditions and to solve Ricci equation. The problem related to Ricci flows was introduced by the following short-time existence theorem:

Theorem. Given a compact and smooth Riemannian manifold $\left(\mathcal{M}^{n}, g_{0}\right)$, there exists a unique smooth solution $g(t)$ defined on a short-time-interval such that $g(0)=g_{0}$.

Another topic connected with Ricci flows remains for us to be done here is to distinguish in which case the long-time existence of Ricci flows is tenable, and the solution of Ricci flows converges to a constant curvature metric. The research in this respect is usually aimed at the positive curvature case. Moreover, study of the singularity [18] of solutions of Ricci flows and the estimation [24] of geometric invariants associated with different pinch-conditions has achieved relatively profound and sufficient development.

Though many scholars have focused their attention on Ricci flows and have arrived at many interesting results $[4,11,12,13,14,15,16]$ since Ricci flows were introduced by R. Hamilton, there are many important and interesting problems still open about Ricci flows such as the stability problem of Ricci flows. In general, this class question can be written as follows.

Let the solution $g(t)$ of Ricci flows with initial value $g_{0}$ converge, and $\tilde{g}_{0}$ belong to a neighborhood of $g_{0}$, then, is it true that the solution $\tilde{g}(t)$ of Ricci flows with initial value $\tilde{g}_{0}$ converges ?

The study (Ye [24], 1993) of stability of Ricci flows makes a start from a metric of constant non-zero sectional curvature, and from replacing the original Ricci flows with the value-normalized Ricci flows

$$
\frac{\partial}{\partial t} g=-2 R c[g]+\frac{2}{n}(\oint R d \mu) g, g(0)=g_{0},
$$

where $\oint R d \mu \doteqdot \frac{\int R d \mu}{\int d \mu}$. Ye [24] also studied that there exists a $C^{2}$-neighborhood $\mathcal{N}\left(g_{0}\right)$ of $g_{0}$ such that, for any $\tilde{g}_{0} \in \mathcal{N}\left(g_{0}\right)$, the solution of Ricci flows $\tilde{g}(t)$ corresponding to $\tilde{g}_{0}$ converges to $g_{0}$ if $g_{0}$ is of a Riemannian-Pinched Einstein metric with non-zero scalar curvature. For the stability of Ricci flows of the flat metric, he did not work out a solution. Following on the heels of Ye's work, C. Guenther etc [10], in 2001, studied the following problems:

(1) The stability of the solution of Ricci flows with flat initial value $g_{0}$;

(2) The stability of the solution of Ricci flows with Ricci flat but not flat initial value metric $g_{0}$;

(3) The stability of Ricci flows with Kähler-Einstein initial value metric $g_{0}$ on $\mathrm{K} 3$;

(4) The stability of the solution of DeTurck flows with non-zero constant curvature initial metric $g_{0}$.

C. Guenther [10] first introduced center manifolds [6] and maximal regularity theory $[17,21]$ to derive the stability of Ricci (DeTurck) flows in constant curvature spaces. The maximal regularity theory says that if $\mathcal{A}$ is a suitable 
quasi-linear differential operator acting on an appropriate function space, and if its linearization $D \mathcal{A}$ at a fixed point has an eigenvalue on the imaginary axis, then the evolution of solutions starting near that fixed point can be described by the presence of exponentially attractive center manifolds.

Since the Ricci flow evolution equation

$$
\frac{\partial}{\partial t} g=-2 R c[g], g(0)=g_{0}
$$

is not a strictly parabolic system, the maximal regularity theory can not be applied directly to it. Thus, one finds a strictly parabolic evolution equation, i.e., the DeTurck equation [7]

$$
\frac{\partial}{\partial t} g=-2 R c[g]-P_{u}(g), g(0)=g_{0}
$$

to replace the Ricci flow equation. As we know that the solution of (1.2) is equivalent to that of (1.1) up to a simple parameter diffeomorphic transform group. Then, one can arrive at the stability of convergent DeTurck flows by virtue of the stability of convergent Ricci flows.

It is well known that C. Guenther [10] studied, by using the indirect approach, the stability of Ricci flows corresponding to initial value metric with non-zero constant curvature, but in this setting the DeTurck flow does not satisfy maximal regularity theory: no matter what $u$ takes, any stable solution to this equation does not exist. Then, one considers the normalized DeTurck equation as follows

$$
\frac{\partial}{\partial t} g=-2 R c[g]-P_{u}(g)+\frac{2}{n}(\oint R d \mu) g, g(0)=g_{0} .
$$

In fact, in this setting, $u=g_{0}$ is a stable solution of (1.3).

Because of the fact that a Riemannian manifold of the quasi-constant curvature is the special quasi-Einstein space [25, 26], we generalized naturally the problem (1.2) to the quasi-constant curvature manifold, i.e., we studied the quasi-DeTurck flows [3] as follows

$$
\frac{\partial}{\partial t} g=-2 R c[g]-P_{u}(g)+2 \frac{R-T}{n-1} g+2 \frac{n T-R}{n-1} \xi \otimes \xi, g(0)=g_{0},
$$

where $\xi$ is of a unit vector field, and $T$ is the Ricci principal curvature corresponding to $\xi$. Notice that the stability here is different from that posed in $[27]$.

Just as we know that the DeTurck flows, given by C. Guenther [10], are in fact obtained by adding $P_{u}(g)$ to Ricci flows such that all the quadratic terms up to Laplace operator vanishes, and thus they shared the same principal symbols with Laplace operator.

Motivated and inspired by the structure of DeTurck flows, in this paper, we consider the same problems posed in [10], but we adopt the other arguments to derive and obtain the similar interesting conclusions, this argument successfully 
avoids DeTurck flows and makes one consider directly Ricci flows. Moreover, we also study quasi-Ricci flows

$$
\frac{\partial}{\partial t} g=-2 R c[g]+2 \frac{R-T}{n-1} g+2 \frac{n T-R}{n-1} \xi \otimes \xi, g(0)=g_{0}
$$

by using the similar argument posed in [3] in this paper.

The organization of this paper is as follows. In Section 2, we will recall and give some necessary notations and terminologies. Section 3 is devoted to the proofs of theorems. In this section we will consider, in view of Killing conditions, the stability of Ricci flows over a constant curvature space, and that of quasi-Ricci flows over a quasi-constant curvature space.

\section{Notations and terminologies}

Let $\mathcal{M}$ be a closed connected smooth manifold, we denote by $S_{2}(\mathcal{M})$ the bundle of symmetric covariant 2-tensors over $\mathcal{M}$, and by $S_{2}^{+}(\mathcal{M})$ the subset of the positive definite tensors. In this setting, a smooth Riemannian metric $g$ is an element of $C^{\infty}\left(S_{2}^{+}(\mathcal{M})\right)$. For convenience, we denote briefly by $\mathcal{S}_{2} \doteqdot$ $C^{\infty}\left(S_{2}(\mathcal{M})\right)$ and $\mathcal{S}_{2}^{+} \doteqdot C^{\infty}\left(S_{2}^{+}(\mathcal{M})\right)$. We also denote by $\mathcal{S}_{2}^{\mu} \doteqdot C^{\infty}\left(S_{2}^{\mu}(\mathcal{M})\right)$ the space of all metrics with the same volume element given by $g$, and by $\mathcal{S}_{2}^{\mu^{+}} \doteqdot C^{\infty}\left(S_{2}^{\mu^{+}}(\mathcal{M})\right)$ the collection of positive definite tensors of $C^{\infty}\left(S_{2}^{\mu}(\mathcal{M})\right)$. Denote by $\Lambda^{p}=\Lambda^{p}\left(T^{*} \mathcal{M}\right)$ the $p$-form bundle on $\mathcal{M}$, and denote by $\Omega^{p}=$ $C^{\infty}\left(\Lambda^{p}\right)$ the differential $p$-form bundle.

Let $\mathcal{D}(\mathcal{M})$ be the smooth diffeomorphic group: $(h, \phi) \mapsto \phi^{*} h$ acting on $\mathcal{S}_{2}^{+}$. On the other hand, it is easy to check that $g$ is of Einstein if and only if $\phi^{*} g$ is of Einstein, where $g$ is a Riemannian metric on $\mathcal{M}$ and its volume form is $d \mu$.

We now define a map $\delta=\delta_{g}: \mathcal{S}_{2} \rightarrow \Omega^{1}$ by

$$
\delta: h \mapsto \delta h=-g^{i j} \nabla_{i} h_{j k} d x^{k}
$$

whose formal adjoint under the $L^{2}$ inner product

$$
(\cdot, \cdot) \doteqdot \int_{M}\langle\cdot, \cdot\rangle d \mu
$$

is the $\operatorname{map} \delta^{*}=\delta_{g}^{*}: \Omega^{1} \rightarrow \mathcal{S}_{2}$ given by

$$
\delta^{*}: \omega \mapsto \frac{1}{2} \mathcal{L}_{\omega^{\sharp}} g=\frac{1}{2}\left(\nabla_{i} \omega_{j}+\nabla_{j} \omega_{i}\right) d x^{i} \otimes d x^{j},
$$

where $\omega^{\sharp}$ is a vector field metrically isomorphic to $\omega$.

By virtue of [7], we define $G: \mathcal{S}_{2}^{+} \times \mathcal{S}_{2} \rightarrow \mathcal{S}_{2}$ as

$$
(g, u) \mapsto G(g, u)=\left(u_{i j}-\frac{1}{2} g^{k l} u_{k l} g_{i j}\right) d x^{i} \otimes d x^{j}
$$

and $P: \mathcal{S}_{2}^{+} \times \mathcal{S}_{2}^{+} \rightarrow \mathcal{S}_{2}$ as

$$
(g, u) \mapsto P(g, u) \doteqdot P_{u}(g)=-2 \delta_{g}^{*}\left(u^{-1} \delta_{g}(G(g, u))\right)
$$


Thus, one can consider the following evolution equation (DeTurck equation)

$$
\frac{\partial}{\partial t} g=-2 R c[g]-P_{u}(g), g(0)=g_{0}
$$

For the sake of convenience, we call $\overline{\mathcal{A}}_{u}(g) g \doteqdot-2 R c[g]-P_{u}(g)$ the DeTurck operator, then, the formula (2.5) can be rewritten as

$$
\frac{\partial}{\partial t} g=-2 R c[g]-P_{u}(g)=\overline{\mathcal{A}}_{u}(g) g, g(0)=g_{0}
$$

The right hand of this equation above can be regarded as the quasi-linear operator with respect to $g$, in fact, we have the following:

Lemma 2.1 ([10]). The DeTurck operator $\overline{\mathcal{A}}_{u}(g) g$, in the local sense, can be written as

$\left(\overline{\mathcal{A}}_{u}(g) g\right)_{i j}=a(x, u, g)_{i j}^{k l p q} \frac{\partial^{2}}{\partial x^{p} \partial x^{q}} g_{k l}+b(x, u, \partial u, g)_{i j}^{k l p} \frac{\partial}{\partial x^{p}} g_{k l}+c(x, u, \partial u)_{i j}^{k l} g_{k l}$, where $a(x, \cdot, \cdot), b(x, \cdot, \cdot, \cdot), c(x, \cdot, \cdot)$ are smooth functions with respect to $x \in \mathcal{M}^{n}$, respectively, and are analytic with respect to the remaining arguments.

Lemma 2.2 ([10]). The right hand of (2.5) and Laplacian operator have the same symbol. Then, for any $u \in \mathcal{S}_{2}^{+}$, the equation $(2.5)$ is strictly parabolic, its unique solution $g$ provides a unique solution $\phi_{t}^{*} g$ of (1.1) with initial value $g_{0}$, where the diffeomorphisms $\phi_{t}$ are generated by integrating the vector field

$$
V^{i} \doteqdot g_{i j} u_{j k}^{-1} g^{k l} g^{p q}\left(\nabla_{p} u_{q l}-\frac{1}{2} \nabla_{l} u_{p q}\right) .
$$

Assume that $(\mathcal{M}, g)$ is a Riemannian manifold, we denote by $\Delta=g^{i j} \nabla_{i} \nabla_{j}$ the Laplace operator. Let $\Delta_{l}$ be the Licherowicz-Laplace operator such that $\Delta_{l}: \mathcal{S}_{2} \rightarrow \mathcal{S}_{2}$ given by

$$
\Delta_{l} h_{j i}=\Delta_{l} h_{j i}+2 R_{j p q i} h^{p q}-R_{j}^{k} h_{k i}-R_{i}^{k} h_{j k} .
$$

Lemma 2.3 ([10]). Let $g \in \mathcal{S}_{2}^{+}, h \in \mathcal{S}_{2}$, and define $H \doteqdot t r_{g} h \doteqdot g^{j i} h_{j i}$, divh $h_{k} \doteqdot$ $\nabla^{p} h_{k p}$. Let $\tilde{g}=g+\epsilon h$, and denote by $\tilde{\Gamma}, \tilde{R}, d \tilde{\mu}$ the Christoffel coefficient, curvature tensor, volume element of $\tilde{g}$, respectively. Then, one arrives at

$$
\begin{aligned}
\left.\frac{\partial}{\partial \epsilon} \tilde{\Gamma}_{i j}^{k}(\tilde{g})\right|_{\epsilon=0} & =\frac{1}{2} g^{k l}\left(\nabla_{i} h_{j l}+\nabla_{j} h_{i l}-\nabla_{l} h_{i j}\right) ; \\
\left.\frac{\partial}{\partial \epsilon} \tilde{R}_{i j k}^{l}(\tilde{g})\right|_{\epsilon=0}= & \frac{1}{2}\left(\nabla_{i} \nabla_{k} h_{j}^{l}-\nabla_{i} \nabla^{l} h_{j k}-\nabla_{j} \nabla_{k} h_{i}^{l}\right. \\
& \left.+\nabla_{j} \nabla^{l} h_{i k}+R_{i j m}^{l} h_{k}^{m}-R^{m}{ }_{i j k} h_{m}^{l}\right) ; \\
\left.\frac{\partial}{\partial \epsilon} d \tilde{\mu}(\tilde{g})\right|_{\epsilon=0}= & \frac{1}{2} H d \mu ; \\
\left.\frac{\partial}{\partial \epsilon} \tilde{g}^{i j}\right|_{\epsilon=0}= & -g^{i k} g^{j l} h_{k l}=-h^{i j} ; \\
\left.\frac{\partial}{\partial \epsilon}\left(\mathcal{L}_{X} \tilde{g}\right)_{i j}\right|_{\epsilon=0}= & X^{k} \nabla_{k} h_{i j}+h_{i k} \nabla_{j} X^{k}+h_{j k} \nabla_{i} X^{k} .
\end{aligned}
$$


Definition 2.1. Let $X=X(g, h)$ be a 1 -form given by $X_{k}=g^{p q} \nabla_{p} h_{q k}-$ $\frac{1}{2} \nabla_{k}\left(g^{p q} h_{p q}\right)$. We say that Ricci flows satisfy Killing conditions if it holds

$$
\left(\mathcal{L}_{X^{\sharp}} g\right)_{j i}=\nabla_{j} X_{i}+\nabla_{i} X_{j}=0 .
$$

Let $\Theta_{0}, \Theta_{1}, \Xi_{0}, \Xi_{1}$ be the Banach spaces such that there holds [2]: $\Xi_{0}=$ $h^{0+\sigma} \supset \Theta_{0}=h^{0+\rho} \supset \Xi_{1}=h^{2+\sigma} \supset \Theta_{1}=h^{2+\rho}$, where $0<\sigma<\rho<1$, $h^{r+\rho}(r \in \mathbb{N}, \rho \in(0,1))$ is the special little Hölder space. Assume that $\|\cdot\|_{r+\rho}$ is the Hölder norm of $C^{r}\left(M, \mathcal{S}_{2}\right)$. Taking $\theta=\frac{\rho-\sigma}{2} \in(0,1)$, by using $[2,8,22]$, one gets $\Theta_{0} \cong\left(\Xi_{0}, \Xi_{1}\right)_{\theta}$ and $\Theta_{1} \cong\left(\Xi_{0}, \Xi_{1}\right)_{1+\theta}$.

For the given $0<\epsilon \ll 1$ and $\frac{1}{2} \leq \beta<\alpha<1$, let

$G_{\beta}=G_{\beta}(u, \epsilon)=\left\{g \in\left(\Theta_{0}, \Theta_{1}\right)_{\beta}: g>\epsilon u\right\}, G_{\alpha}=G_{\alpha}(u, \epsilon)=G_{\beta} \cap\left(\Theta_{0}, \Theta_{1}\right)_{\alpha}$, where $g>\epsilon u$ implies that it holds $g(X, X)>\epsilon$ for any $X$ satisfying $|X|_{u}^{2}=1$.

Moreover, for any $g \in G_{\beta}, \overline{\mathcal{A}}_{u}(g)$ can be regarded as a linear operator acting on $h^{2+\sigma}$. Denote by $\overline{\mathcal{A}}_{\Xi_{1}}(g): \Xi_{1} \subseteq \Xi_{0} \rightarrow \Xi_{0}$ the unbounded linear operator on $\Xi_{0}$, its dense domain $D\left(\overline{\mathcal{A}}_{\Xi_{1}}(g)\right)=\Xi_{1}$. Make corresponding changes and denote by $\overline{\mathcal{A}}_{\Theta_{1}}(g): \Theta_{1} \subseteq \Theta_{0} \rightarrow \Theta_{0}$ the unbounded linear operator whose dense domain $D\left(\overline{\mathcal{A}}_{\Theta_{1}}(g)\right)=\Theta_{1}$. At the same time, the functions $g \mapsto \overline{\mathcal{A}}_{\Theta_{1}}(g)$ and $g \mapsto \overline{\mathcal{A}}_{\Xi_{1}}(g)$ define the analytic maps given by $G_{\alpha} \rightarrow L\left(\Theta_{1}, \Theta_{0}\right), G_{\beta} \rightarrow L\left(\Xi_{1}, \Xi_{0}\right)$, where $L\left(\Theta_{1}, \Theta_{0}\right)$ is the vector space of all bounded linear operators from $\Theta_{1}$ to $\Theta_{0}$, and for any $g \in G_{\beta}, \overline{\mathcal{A}}_{u}(g)$ is the minimal generator of a strongly continuous analytic semigroup.

Theorem 2.1 ([10, 17, 21]). Let $\Theta_{1} \subset \Theta_{0}$ be a continuous dense inclusion of a Banach space. For a given $0<\beta<\alpha<1$, suppose that $\Theta_{\alpha}$ and $\Theta_{\beta}$ are the corresponding interpolation space. For the following equation

$$
\frac{\partial}{\partial t} g=\overline{\mathcal{A}}(g) g, g(0)=g_{0},
$$

where $\overline{\mathcal{A}}(\cdot) \in C^{k}\left(G_{\beta}, L\left(\Theta_{1}, \Theta_{0}\right)\right)$, and $k$ is a positive integer, $G_{\beta} \subset \Theta_{\beta}$ is an open subset. Assume that there exist a pair Banach space $\Xi_{0} \supset \Xi_{1}$ and a prolongation $\tilde{\mathcal{A}}(\cdot)$ of $\overline{\mathcal{A}}(\cdot)$ to domain $D(\tilde{\mathcal{A}}(\cdot))$ that are dense in $\Xi_{0}$. In addition, for any $g \in G_{\alpha}=G_{\beta} \cap \Theta_{\alpha}$, there holds

- $\tilde{\mathcal{A}}(g) \in L\left(\Xi_{1}, \Xi_{0}\right)$ generates a strongly continuous semigroup on $L\left(\Xi_{0}, \mathbb{R}\right) \doteqdot$ $L\left(\Xi_{0}\right)$;

- $\Theta_{0} \cong\left(\Xi_{0}, D(\tilde{\mathcal{A}}(g))\right)_{\theta}, \Theta_{1} \cong\left(\Xi_{0}, D(\tilde{\mathcal{A}}(g))\right)_{1+\theta}, \theta \in(0,1)$, where $(\cdot, \cdot)_{\theta}$ are the continuous interpolations $[8,22]$;

- $\overline{\mathcal{A}}(g)$ is identical to $\tilde{\mathcal{A}}(g)$ on $D(\overline{\mathcal{A}}) \subset \Theta_{0}$;

- $\Xi_{1} \hookrightarrow \Theta_{\beta} \hookrightarrow \Xi_{0}$ is a continuous dense inclusion and there exists $c>0, \delta \in$ $(0,1)$ such that for any $\eta \in \Xi_{1}$ there holds

$$
\|\eta\|_{\Theta_{\beta}} \leq c\|\eta\|_{\Xi_{0}}^{1-\delta}\|\eta\|_{\Xi_{1}}^{\delta} .
$$

Let $\hat{g} \in G_{\alpha}$ be a fixed point of (1.2) and the spectral decompositions ([1]) $\sum$ of the linearization operator $\left.D \overline{\mathcal{A}}\right|_{g}$ be of $\sum=\sum_{s} \cup \sum_{c u}$, where $\sum_{s} \subset\{z$ : $\operatorname{Re} z<0\}, \sum_{c u} \subset\{z: \operatorname{Re} z \geq 0\}$ and $\sum_{c u} \cap i \mathbb{R} \neq \emptyset$, then it holds 
(1) If one denotes by $S(\lambda)$ the eigenspace of $\lambda \in \sum_{c u}$, then $\Theta_{\alpha}$ admits the decomposition $\Theta_{\alpha}=\Theta_{\alpha}^{s} \oplus \Theta_{\alpha}^{c u}$ for all $\alpha \in[0,1]$, where $\Theta_{\alpha}^{c u}=\bigoplus_{\lambda \in \sum_{c u}} S(\lambda)$;

(2) For any $r \in \mathbb{N}$, there exists $d_{r}>0$ such that for all $d \in\left(0, d_{r}\right]$, there is a bounded $C^{r}$ map $\varphi=\varphi_{d}^{r}: B\left(\Theta_{1}^{c u}, \hat{g}, d\right) \rightarrow \Theta_{1}^{s}$ with $\varphi(\hat{g})=0$ and $D \varphi(\hat{g})=0$. The image of $\varphi$ lies in the closed ball $\bar{B}\left(\Theta_{1}^{s}, \hat{g}, d\right)$, and its graph is a $C^{r}$ manifold $\mathcal{M}_{\text {loc }}^{c u} \doteqdot\left\{(\gamma, \varphi(\gamma)): \gamma \in B\left(\Theta_{1}^{c u}\right), \hat{g}, d\right\} \subset \Theta_{1}$ satisfying the following

$$
T_{\hat{g}} \mathcal{M}_{l o c}^{c u} \cong \Theta_{1}^{c u} .
$$

If $\sum_{c u} \subset i \mathbb{R}$, we call $\mathcal{M}_{\text {loc }}^{c u}$ a local center manifold ([6]) and a local center unstable manifold otherwise;

(3) For all $\alpha \in(0,1)$, there are constants $C_{\alpha}>0$ independent of $\hat{g}$ and constant $\omega>0$ and $\hat{d} \in\left(0, d_{0}\right]$ such that for each $d \in(0, \hat{d}]$, one arrives at

$$
\left\|\pi^{s} g(t)-\varphi\left(\pi^{c u} g(t)\right)\right\|_{\Theta_{1}} \leq \frac{C_{\alpha}}{t^{1-\alpha}} e^{-\omega t}\left\|\pi^{s} g(0)-\varphi\left(\pi^{c u} g(0)\right)\right\|_{\Theta_{\alpha}}
$$

for all solutions $g(t)$ with $g(0) \in B\left(\Theta_{\alpha}, \hat{g}, d\right)$ and all times $t \geq 0$ such that the solution $g(t)$ remains in $B\left(\Theta_{\alpha}, \hat{g}, d\right)$. Where $\pi^{s}, \pi^{c u}$ say the projections from $B\left(\Theta_{\alpha}, \hat{g}, d\right)$ onto $\Theta_{\alpha}^{s}$, and $\Theta_{\alpha}^{c u}$, respectively.

\section{Main theorems and proofs}

For convenience, we first state some well known notations and terminologies in the first two subsections.

\subsection{Ricci operator}

Let $V, W$ be two vector bundles over a manifold $\mathcal{M}^{n}$. Assume that $\mathcal{L}$ : $C^{\infty}(V) \rightarrow C^{\infty}(W)$ is a linear differential operator with order $k$. Denote by $\mathcal{L}(\nu) \doteqdot \sum_{|\alpha| \leq k} \mathcal{L}_{\alpha} \partial^{\alpha} \nu$, where $\mathcal{L}_{\alpha} \in \operatorname{hpm}(V, W)$ is a bundle homomorphism for each multi-index $\alpha$. If $\xi \in C^{\infty}\left(T^{*} M^{n}\right)$, then we call $\sigma[\mathcal{L}](\xi)=$ $\sum_{|\alpha| \leq k} \mathcal{L}_{\alpha}\left(\Pi_{j} \xi^{\alpha j}\right)$ the total symbol of $\mathcal{L}$ in the direction $\xi$. We also call $\hat{\sigma}[\mathcal{L}](\xi)=\sum_{|\alpha|=k} \mathcal{L}_{\alpha}\left(\Pi_{j} \xi^{\alpha j}\right)$ the principal symbol of $\mathcal{L}$ in the direction $\xi$.

A linear partial differential operator $\mathcal{L}$ is said to be elliptic if its principal symbol $\hat{\sigma}[\mathcal{L}](\xi)$ is an isomorphism where $\xi \neq 0$. A nonlinear operator $L$ is said to be elliptic if its linearization $D L$ is elliptic.

For Ricci flows, we use Lemma 2.3 and write down the following

$D R_{j i}=\frac{1}{2}\left(\Delta h_{j i}+\nabla_{j} \nabla_{i} H-\nabla_{j} \operatorname{div} h_{i}-\nabla_{i} \operatorname{div} h_{j}+2 R_{j p q i} g^{p q}-R_{j l} h_{i}^{l}-R_{i l} h_{j}^{l}\right)$, then, one gets

$$
-2[D(R c)]_{j i}=\Delta h_{j i}-\nabla_{j} X_{i}-\nabla_{i} X_{j}+S_{j i},
$$

where $X_{j}$ is of the 1 -form defined by $X_{j}=g^{p q} \nabla_{p} h_{q j}-\frac{1}{2} \nabla_{j}\left(g^{p q} h_{p q}\right)$, and $S_{j i}=2 R_{j p q i} g^{p q}-R_{j l} h_{i}^{l}-R_{i l} h_{j}^{l}$.

In other words, one arrives at the following

$$
\hat{\sigma}[-2 D(R c)](\xi)(h)=|\xi|^{2} h .
$$


This implies that the linearized Ricci flow in view of Killing 1-form is elliptic. In this setting, we call $\mathcal{A}(g) g=-2 R c[g]+\frac{2}{n}(\oint R d \mu) g$ the Ricci operator. Then, the volume-normalized Ricci flow [24] can be rewritten as

$$
\frac{\partial}{\partial t} g=\mathcal{A}(g) g, g(0)=g_{0} .
$$

\subsection{Linearized Ricci operator}

In this subsection, we will pay our attention to the linearization of the Ricci operator.

Lemma 3.2.1. Assume that $\mathcal{M}^{n}$ is of a closed compact constant curvature space. Then the linearized Ricci operator $\mathcal{A}(g) g$, satisfying Killing conditions, at $g_{0}$ is as follows

$$
\left[\left.(D \mathcal{A}(g) g)\right|_{g_{0}}\right]_{j i}=\Delta h_{j i}+2 R_{j p q i} h^{p q}-\frac{2 R}{n^{2}}\left[\frac{\int_{\mathcal{M}_{n}} H d \mu}{\int_{\mathcal{M}^{n}} d \mu}\right] g_{j i},
$$

where $H=g^{j i} h_{j i}$.

Proof. In fact, by using Definition, one has

$$
\left.\left[D \mathcal{A}_{u}(g)\right]\right|_{g_{0}} h=-\left.2 D R c\right|_{g_{0}}+\left.\frac{2}{n} D(\oint R d \mu)\right|_{g_{0}} g+\frac{2}{n} \oint H d \mu h .
$$

According to Lemma 2.3, one arrives at

$$
\begin{aligned}
\left(-\left.2 D R c\right|_{g_{0}} h\right)_{j i}= & \Delta h_{j i}-\nabla_{j}\left(g^{p q} \nabla_{p} h_{p i}\right)-\nabla_{i}\left(g^{p q} \nabla_{p} h_{p j}\right) \\
& +\nabla_{j} \nabla_{i}\left(g^{p q} h_{p q}\right)+2 g^{p q} R_{p j i}^{r} h_{r q}-g^{p q} R_{j p} h_{i q}-g^{p q} R_{i p} h_{j q} .
\end{aligned}
$$

Since $\left(\mathcal{M}^{n}, g_{0}\right)$ is of an Einstein manifold, in addition, by using Killing hypothesis, we get

$$
\begin{aligned}
\left(-\left.2 D R c\right|_{g_{0}} h\right)_{j i} & =\Delta h_{j i}+2 g^{p q} R^{r}{ }_{j i} h_{r q}-\frac{2 R}{n} h_{j i}, \\
\frac{2}{n} D(\oint R d \mu) g & =\frac{2}{n}\left[\oint\left(\frac{1}{2}(R-\oint R d \mu) H-\langle R c, h\rangle\right) d \mu\right] g \\
& =-\frac{2}{n}[\oint\langle R c, h\rangle d \mu] g .
\end{aligned}
$$

Thus, we have

$$
\begin{aligned}
& \left(-\left.2 D \mathcal{A}_{u}(g)\right|_{g_{0}} h\right)_{j i} \\
= & \Delta h_{j i}+2 R_{j p q i} h_{p q}-\frac{2 R}{n} h_{j i}-\frac{2}{n}[\oint\langle R c, h\rangle d \mu] g_{j i}+\frac{2 R}{n} h_{j i} \\
= & \Delta h_{j i}+2 R_{j p q i} h^{p q}-\frac{2 R}{n^{2}}\left[\frac{\int_{\mathcal{M}_{n}} H d \mu}{\int_{\mathcal{M}_{n}} d \mu}\right] g_{j i} .
\end{aligned}
$$

This ends the proof of Lemma 3.2.1. 
Notice that $g_{0}$ is a stable point of (1.5) for quasi-constant curvature spaces [3, 24]. Then we can state and derive the main conclusions in the next subsection.

\subsection{Main theorems and proofs}

Theorem 3.3.1. Let $\mathcal{M}^{n}$ be a compact manifold of constant curvature, and $\|R m\| \leq \Lambda$, where $\Lambda=\inf _{h}\left\{\frac{\int_{\mathcal{M}^{n}}\|\nabla h\|^{2}}{\int_{\mathcal{M}^{n}}\|h\|^{2}}\right\}, h$ is of a $(0,2)$-type tensor. Assume that Ricci flows satisfy Killing condition, and for a fixed $\rho \in(0,1), \Theta$ is of a closure of $\mathcal{S}_{2}^{\mu}\left(\supset \mathcal{S}_{2}^{\mu^{+}}\right)$in the sense of $\|\cdot\|_{2+\rho}$. Then it holds the following

(1) $T_{g_{0}} \mathcal{S}_{2}^{\mu^{+}} \cong \Theta$ has a decomposition: $T_{g_{0}} \mathcal{S}_{2}^{\mu^{+}}=\Theta^{s} \oplus \Theta^{c}$;

(2) For each $r \in \mathbb{N}$, there exists a $C^{r}$-center manifold $\mathcal{M}_{\text {loc }}^{c}$ that is tangential to $\Theta^{c}$ in an neighborhood $\mathcal{O}_{r}$ of $g_{0}$ on $\Theta$ and is locally invariant for solutions of (3.1.3) as long as they remain in $\mathcal{O}_{r}$;

(3) There exist positive constants $C$ and $\omega$, and neighborhoods $\mathcal{O}_{r}^{\prime}$ of $g_{0}$ in $\Theta$ such that

$$
\left\|\pi^{s} \tilde{g}(t)-\varphi\left(\pi^{c} \tilde{g}(t)\right)\right\|_{2+\rho} \leq C e^{-\omega t}\left\|\pi^{s} \tilde{g}(0)-\varphi\left(\pi^{c} \tilde{g}(0)\right)\right\|_{2+\rho}
$$

for all solution $\tilde{g}(t)$ of (3.1.3) and all times $t \geq 0$ such that $\tilde{g}(t) \in \mathcal{O}_{r}^{\prime}$.

Proof. We take $\mathcal{S}_{2}^{\mu} \doteqdot C^{\infty}\left(S_{2}^{\mu}(\mathcal{M})\right)$ as the space of all metrics with the same volume element given by $g_{0}$. By [19], one knows that the elements in $\mathcal{S}_{2}^{+}$can be changed into those in $\mathcal{S}_{2}^{\mu}$ by using homothetic deformations and the tangent space $T \mathcal{S}_{2}^{\mu}$ of $\mathcal{S}_{2}^{\mu}$ consists of all zero-trace elements in $\mathcal{S}_{2}$. Then, on $T \mathcal{S}_{2}^{\mu}$, there holds $H=0$. On the other hand, it is well known that $g_{0}$ is a stable point of (3.1.3). Then, formula (3.2.1) can be simplified as

$$
\frac{\partial}{\partial t} h_{j i}=\mathcal{L} h_{j i}=\Delta h_{j i}+2 R_{j p q i} h^{p q}
$$

Thus, we know

$$
\begin{aligned}
\int_{\mathcal{M}^{n}} R_{j p q i} h^{p q} h_{i j} d \mu & =\int_{\mathcal{M}^{n}} R_{j p q i} g^{p l} g^{q m} h_{l m} h_{j i} d \mu \\
& =\int_{\mathcal{M}^{n}} R_{j i}^{l m} h_{l m} h_{j i} d \mu \\
& \leq \int_{\mathcal{M}^{n}} \Lambda h_{l m} h_{j i} d \mu \\
& \leq \Lambda\left(\int_{\mathcal{M}^{n}} h_{l m}^{2} d \mu\right)^{\frac{1}{2}}\left(\int_{\mathcal{M}^{n}} h_{j i}^{2} d \mu\right)^{\frac{1}{2}} \leq \Lambda\|h\|_{L^{2}}^{2} .
\end{aligned}
$$

This implies that $(\mathcal{L} h, h)=-2 \int_{\mathcal{M}^{n}}(\nabla h)^{2} d \mu+2 \Lambda\|h\|_{L^{2}}^{2} \leq 0$, where $h \in \mathcal{S}_{2}^{0}$ is of an non-zero element. Considering the operator $\mathcal{L}$ acting on $\mathcal{S}_{2}^{\mu}$, it is not hard to see by virtue of [10] that Theorem 3.3.1 is tenable. 
Theorem 3.3.2. Assume that $\left(\mathcal{M}^{n}, g_{0}\right)$ is of a quasi-constant curvature space, $\|R m\| \leq \Lambda$, where $2 \Lambda=\inf _{h}\left\{\frac{\int_{\mathcal{M}}\|\nabla h\|^{2}}{\int_{\mathcal{M}}\|h\|^{2}}\right\}, \xi$ is a unit vector field and its corresponding Ricci principal curvature $T$ satisfies $T \geq n-1$. For a fixed $\rho \in(0,1)$, let $\Theta$ be a closure of $S_{2}^{\mu}$ in the sense of $\|\cdot\|_{2+\rho}$. In addition, the quasi-Ricci flow equation (1.5) satisfies Killing conditions, then it holds

(1) $T_{g_{0}} S_{2}^{\mu^{+}} \cong \Theta$ has the following decomposition: $T_{g_{0}} S_{2}^{\mu^{+}}=\Theta^{s} \oplus \Theta^{c}$

(2) There exists a constant $d_{0}>0$ such that for all $d \in\left(0, d_{0}\right]$, there is a bounded $C^{\infty}$ map $\psi: B\left(\Theta^{c}, g_{0}, d\right) \rightarrow \Theta^{s}$ satisfying $\psi\left(g_{0}\right)=0, D \psi\left(g_{0}\right)=0$, the image of $\psi$ dependent on the closed ball $\bar{B}\left(\Theta^{s}, g_{0}, d\right)$ and its graph $M_{\text {loc }}^{c}=$ $\left\{(\gamma, \psi(\gamma)): \gamma \in B\left(\Theta^{c}, g_{0}, d\right)\right\} \subset \Theta_{1}$ satisfying $T_{g_{0}} M_{l o c}^{c} \cong \Theta^{c}$

(3) There are constants $C>0, \omega>0$ and $d_{*} \in\left(0, d_{0}\right]$ such that for each $d \in\left(0, d_{*}\right]$, one arrives at

$$
\left\|\pi^{s} \tilde{g}(t)-\psi\left(\pi^{c} \tilde{g}(t)\right)\right\|_{2+\rho} \leq C e^{-\omega t}\left\|\pi^{s} \tilde{g}(0)-\psi\left(\pi^{c} \tilde{g}(0)\right)\right\|_{2+\rho}
$$

for all solutions $\tilde{g}(t)$ of the quasi-Ricci flow (1.5) with $\tilde{g}(0) \in B\left(\Theta, g_{0}, d\right)$ and all times $t \geq 0$, where $\pi^{s}, \pi^{c}$ denote the projections onto $\Theta^{s}, \Theta^{c}$ respectively.

Remark 3.3.1. Theorem 3.3.2 is a generalization of Theorem 3.1 in [3]. The symmetry condition $[1,3,23]$ of $h$ with respect to covariant derivative in Theorem 3.1 is eliminated here.

Proof. Considering the linearization of the right-hand (Denoted also by $\mathcal{A}(g) g$ ) of (1.5) at $g_{0}$, one has

$$
\begin{aligned}
\frac{\partial}{\partial t} h_{j i} & =\left(\left.D\left(\mathcal{A}_{u}(g)\right)\right|_{g_{0}} h\right)_{j i} \\
& =-2\left(\left.D R c\right|_{g_{0}} h\right)_{j i}+\left.2 D\left(\frac{R-T}{n-1} g_{j i}+\frac{n T-R}{n-1} \xi_{i} \xi_{j}\right)\right|_{g_{0}} .
\end{aligned}
$$

By using Lemma 2.3 and [5], we get the following

$$
\begin{aligned}
\left.D R\right|_{g_{0}}= & -\triangle H+\nabla^{p} \nabla^{q} h_{p q}-\langle h, R c\rangle \\
\left.D T\right|_{g_{0}}= & D\left(\xi^{i} \xi^{j} R_{j i}\right)=-m^{i} \xi^{j} R_{i j}-\xi^{i} m^{j} R_{i j}+\xi^{i} \xi^{j} D R_{i j} \\
= & -m^{i} \xi^{j} R_{i j}-\xi^{i} m^{j} R_{i j} \\
& +\frac{1}{2} \xi^{i} \xi^{j}\left(\nabla^{p} \nabla_{i} h_{j p}+\nabla^{p} \nabla_{j} h_{i p}-\triangle h_{i j}-\nabla_{i} \nabla_{j} H\right),
\end{aligned}
$$

where $m$ is of the variational of $\xi$. Thus, we know

$$
\begin{aligned}
& \left.2 D\left(\frac{R-T}{n-1} g_{i j}\right)\right|_{g_{0}} \\
= & \frac{2}{n-1} D(R-T) g_{i j}+\frac{2}{n-1}(R-T) h_{i j} \\
= & \frac{2}{n-1}\left(-\triangle H+\nabla^{p} \nabla^{q} h_{p q}-\langle h, R c\rangle\right) g_{i j}+\frac{2}{n-1}\left[m^{k} \xi^{l} R_{k l}+\xi^{k} m^{l} R_{k l}\right.
\end{aligned}
$$




$$
\left.-\frac{1}{2} \xi^{k} \xi^{l}\left(\nabla^{p} \nabla_{k} h_{l p}+\nabla^{p} \nabla_{l} h_{k p}-\triangle h_{k l}-\nabla_{k} \nabla_{l} H\right)\right] g_{i j}+\frac{2}{n-1}(R-T) h_{i j}
$$

and

$$
\begin{aligned}
& \left.2 D\left(\frac{n T-R}{n-1} \xi_{i} \xi_{j}\right)\right|_{g_{0}} \\
= & \frac{2}{n-1} D(n T-R) \xi_{i} \xi_{j}+\frac{2}{n-1}(n T-R) D\left(\xi_{i} \xi_{j}\right) \\
= & \frac{2 n D T}{n-1} \xi_{i} \xi_{j}-\frac{2 D R}{n-1} \xi_{i} \xi_{j}+\frac{2}{n-1}(n T-R) D\left(\xi_{i} \xi_{j}\right) \\
= & \frac{2 n}{n-1}\left[-m^{k} \xi^{l} R_{k l}-\xi^{k} m^{l} R_{k l}\right. \\
& \left.+\frac{1}{2} \xi^{k} \xi^{l}\left(\nabla^{p} \nabla_{k} h_{l p}+\nabla^{p} \nabla_{l} h_{k p}-\triangle h_{k l}-\nabla_{k} \nabla_{l} H\right)\right] \xi_{i} \xi_{j} \\
& -\frac{2 \xi_{i} \xi_{j}}{n-1}\left(-\triangle H+\nabla^{p} \nabla^{q} h_{p q}-\langle h, R c\rangle\right)+\frac{2}{n-1}(n T-R) D\left(\xi_{i} \xi_{j}\right) .
\end{aligned}
$$

Since $\mathcal{M}$ is a quasi-constant curvature space, in addition, by using [5] and by a direct computation, then we have

$$
\begin{aligned}
& -2[D(R c)(h)]_{j i} \\
= & \triangle h_{j i}+2 R_{j p q i} h^{p q}-R_{j l} h_{i}^{l}-R_{i l} h_{j}^{l}+\nabla_{j} \nabla_{i} H-\nabla_{j} \operatorname{div} h_{i}-\nabla_{i} \operatorname{div} h_{j} \\
= & \triangle h_{j i}+2 R_{i p q j} h^{p q}-2\left(\frac{R-T}{n-1}\right) h_{j i}-\left(\frac{n T-R}{n-1}\right)\left(\xi_{i} \xi_{k} h_{j}^{k}+\xi_{j} \xi_{k} h_{i}^{k}\right) \\
& +\nabla_{j} \nabla_{i} H-\nabla_{j} \operatorname{div} h_{i}-\nabla_{i} \operatorname{div} h_{j} \\
= & \triangle h_{j i}+2 R_{i p q j} h^{p q}-\left(\mathcal{L}_{X^{\sharp}} g\right)_{j i}-2\left(\frac{R-T}{n-1}\right) h_{j i}-\left(\frac{n T-R}{n-1}\right)\left(\xi_{i} \xi_{k} h_{j}^{k}+\xi_{j} \xi_{k} h_{i}^{k}\right),
\end{aligned}
$$

where $X=X(g, h)$ is of 1 -form defined as $X_{k}=g^{p q} \nabla_{p} h_{q k}-\frac{1}{2} \nabla_{k}\left(g^{p q} h_{p q}\right)$.

From formulae (3.3.1), (3.3.2) and (3.3.3), and notice that $2 m_{i} \xi^{i}=-\xi^{i} \xi^{j} h_{i j}$, then we obtain

$$
\begin{aligned}
\frac{\partial}{\partial t} h_{j i}= & \frac{2}{n-1}\left(-\triangle H+\nabla^{p} \nabla^{q} h_{p q}-\langle h, R c\rangle\right) g_{i j}+\frac{2}{n-1}\left[m^{k} \xi^{l} R_{k l}+\xi^{k} m^{l} R_{k l}\right. \\
& \left.-\frac{1}{2} \xi^{k} \xi^{l}\left(\nabla^{p} \nabla_{k} h_{l p}+\nabla^{p} \nabla_{l} h_{k p}-\triangle h_{k l}-\nabla_{k} \nabla_{l} H\right)\right] g_{i j} \\
& +\frac{2 n}{n-1}\left[-m^{k} \xi^{l} R_{k l}-\xi^{k} m^{l} R_{k l}+\frac{1}{2} \xi^{k} \xi^{l}\left(\nabla^{p} \nabla_{k} h_{l p}+\nabla^{p} \nabla_{l} h_{k p}\right.\right. \\
& \left.\left.-\triangle h_{k l}-\nabla_{k} \nabla_{l} H\right)\right] \xi_{i} \xi_{j}-\frac{2 \xi_{i} \xi_{j}}{n-1}\left(-\triangle H+\nabla^{p} \nabla^{q} h_{p q}-\langle h, R c\rangle\right) \\
& +\triangle h_{j i}+2 R_{i p q j} h^{p q}-\left(\mathcal{L}_{X^{\sharp}} g\right)_{j i} \\
= & \triangle h_{j i}+2 R_{i p q j} h^{p q}-\left(\mathcal{L}_{X^{\sharp}} g\right)_{j i}
\end{aligned}
$$




$$
\begin{aligned}
& +\frac{2}{n-1}\left(-\Delta H+\nabla^{p} \nabla^{q} h_{p q}-\frac{R-T}{n-1} H-\frac{n T-R}{n-1} h^{p q} \xi_{p} \xi_{q}\right)\left(g_{j i}-\xi_{j} \xi_{i}\right) \\
& +\frac{\xi^{k} \xi^{l}}{n-1}\left(-2 h_{k l}+\nabla^{p} \nabla_{k} h_{l p}+\nabla^{p} \nabla_{l} h_{k p}-\Delta h_{k l}-\nabla_{k} \nabla_{l} H\right)\left(g_{j i}-n \xi_{j} \xi_{i}\right) .
\end{aligned}
$$

Adopting similar methods in Theorem 3.3.1, we now take $\mathcal{S}_{2}^{\mu} \doteqdot C^{\infty}\left(S_{2}^{\mu}(\mathcal{M})\right)$ as the space of all metrics with the same volume element given by $g_{0}$, and by [19], one knows that the elements in $\mathcal{S}_{2}^{+}$can be changed into those in $\mathcal{S}_{2}^{\mu}$ by using homothetic deformations, and the tangent space $T \mathcal{S}_{2}^{\mu}$ of $\mathcal{S}_{2}^{\mu}$ consists of all trace-zero elements in $\mathcal{S}_{2}$. Then, on $T \mathcal{S}_{2}^{\mu}$, there holds $H=0$. On the other hand, we substitute the quasi-constant curvature tensor, Killing conditions into (3.3.4), and then, we arrive at

$$
\begin{aligned}
\frac{\partial}{\partial t} h_{i j}= & \frac{2}{n-1}\left(\nabla^{p} \nabla^{q} h_{p q}-\frac{n T-R}{n-1} h^{p q} \xi_{p} \xi_{q}\right)\left(g_{i j}-\xi_{i} \xi_{j}\right) \\
& +\frac{\xi^{k} \xi^{l}}{n-1}\left(-2 h_{k l}+\nabla^{p} \nabla_{k} h_{l p}+\nabla^{p} \nabla_{l} h_{k p}-\triangle h_{k l}\right)\left(g_{i j}-n \xi_{i} \xi_{j}\right) \\
& +\triangle h_{i j}-\frac{2(R-2 T)}{(n-1)(n-2)} h_{i j} \\
& +\frac{2(n T-R)}{(n-1)(n-2)}\left(g_{i j} \xi_{p} \xi_{q} h^{p q}-g_{i q} \xi_{p} \xi_{j} h^{p q}-g_{p j} \xi_{i} \xi_{q} h^{p q}\right) .
\end{aligned}
$$

Considering the acting on equation (3.3.5) with $\xi^{i}$ and $\xi^{j}$, we have

$$
\xi^{i} \xi^{j}\left(\frac{\partial}{\partial t} h_{i j}-2 \triangle h_{i j}-2 h_{i j}+\frac{2 T}{n-1} h_{i j}+\nabla^{p} \nabla_{i} h_{j p}+\nabla^{p} \nabla_{j} h_{i p}\right)=0 .
$$

Since $\xi$ is of arbitrary, this implies that

$$
\frac{\partial}{\partial t} h_{i j}-2 \triangle h_{i j}-2 h_{i j}+\frac{2 T}{n-1} h_{i j}+\nabla^{p} \nabla_{i} h_{j p}+\nabla^{p} \nabla_{j} h_{i p}=0 .
$$

By using Killing conditions, we compute attentively and simplify (3.3.6) as follows

$$
\begin{aligned}
\frac{\partial}{\partial t} h_{i j}= & 2 \triangle h_{i j}+2 h_{i j}-\frac{2 T}{n-1} h_{i j} \\
& +g^{t p}\left(R^{k}{ }_{t i p} h_{j k}+R^{k}{ }_{t j p} h_{i k}+R^{k}{ }_{t i j} h_{k p}+R^{k}{ }_{t j i} h_{k p}\right) \\
= & 0 .
\end{aligned}
$$

According to the proof of Theorem 3.3.1, it is not hard to derive that there holds

$$
\begin{aligned}
(\mathcal{L} h, h) & \leq 2 \int_{\mathcal{M}^{n}} \triangle h \cdot h d \mu+2 \int_{\mathcal{M}^{n}}\left(1-\frac{T}{n-1}\right) h^{2} d \mu+4 \Lambda\|h\|_{L^{2}}^{2} \\
& \leq 2\left(\int_{\mathcal{M}^{n}} \nabla(\nabla h \cdot h) d \mu-\int_{\mathcal{M}^{n}}(\nabla h)^{2} d \mu\right)
\end{aligned}
$$




$$
\begin{aligned}
& +2 \int_{\mathcal{M}^{n}}\left(1-\frac{T}{n-1}\right) h^{2} d \mu+4 \Lambda\|h\|_{L^{2}}^{2} \\
\leq & -2 \int_{\mathcal{M}^{n}}(\nabla h)^{2} d \mu+4 \Lambda\|h\|_{L^{2}}^{2}-2 \int_{\mathcal{M}^{n}}\left(\frac{T}{n-1}-1\right) h^{2} d \mu,
\end{aligned}
$$

where $h \in \mathcal{S}_{2}^{0}$ is of a non-zero element. Considering the operator $\mathcal{L}$ acting on $\mathcal{S}_{2}^{\mu}$, it is not hard to see by virtue of [10] that Theorem 3.3.2 is tenable.

Acknowledgments. The first author would like to thank Professors H. Z. Li, Z. Z. Sun, and H. Z. Song for their encouragement and guidance.

\section{References}

[1] M. Berger and D. Ebin, Some decompositions of the space of symmetric tensors on a Riemannian manifold, J. Differential Geometry 3 (1969), 379-392.

[2] P. Butzer and H. Johnen, Lipschitz spaces on compact manifolds, J. Functional Analysis 7 (1971), 242-266.

[3] Q. H. Cai and P. B. Zhao, Stability of quasi-DeTurck flows in Riemannian manifolds of quasi-constant sectional curvatures, Chinese Ann. Math. Ser. A 29 (2008), no. 1, 97-106.

[4] H. D. Cao and B. Chow, Recent developments on the Ricci flow, Bull. Amer. Math. Soc. (N.S.) 36 (1999), no. 1, 59-74.

[5] B. Chow and D. Knopf, The Ricci flow: An introduction, Mathematical Surveys and Monographs, 110. American Mathematical Society, Providence, RI, 2004.

[6] G. Da Prato and A. Lunardi, Stability, instability and center manifold theorem for fully nonlinear autonomous parabolic equations in Banach space, Arch. Rational Mech. Anal. 101 (1988), no. 2, 115-141.

[7] M. DeTurck, Deforming metrics in the direction of their Ricci tensors, J. Differential Geom. 18 (1983), no. 1, 157-162.

[8] G. Dore and A. Favini, On the equivalence of certain interpolation methods, Boll. Un. Mat. Ital. B (7) 1 (1987), no. 4, 1227-1238.

[9] J. Eells and J. Sampson, Harmonic mappings of Riemannian manifolds, Amer. J. Math. 86 (1964), 109-160.

[10] C. Guenther, J. Isenberg, and D. Knopf, Stability of the Ricci flow at Ricci-flat metrics, Comm. Anal. Geom. 10 (2002), no. 4, 741-777.

[11] R. Hamilton, The inverse function theorem of Nash and Moser, Bull. Amer. Math. Soc. (N.S.) 7 (1982), no. 1, 65-222.

[12] - Three-manifolds with positive Ricci curvature, J. Differential Geom. 17 (1982), no. 2, 255-306.

[13] - Four-manifolds with positive curvature operator, J. Differential Geom. 24 (1986), no. 2, 153-179.

[14] - The Ricci flow on surfaces, Mathematics and general relativity (Santa Cruz, CA, 1986), 237-262, Contemp. Math., 71, Amer. Math. Soc., Providence, RI, 1988.

[15] _ The formation of singularities in the Ricci flow, Surveys in differential geometry, Vol. II (Cambridge, MA, 1993), 7-136, Int. Press, Cambridge, MA, 1995.

[16] - Non-singular solutions of the Ricci flow on three-manifolds, Comm. Anal. Geom. 7 (1999), no. 4, 695-729.

[17] D. Henry, Geometric Theorem of Semilinear Parabolic Equations, Springer Verlag, Berlin, 1981.

[18] J. Isenberg and M. Jackson, Ricci flow of locally homogeneous geometries on closed manifolds, J. Differential Geom. 35 (1992), no. 3, 723-741. 
[19] J. Moser, On the volume elements on a manifold, Trans. Amer. Math. Soc. 120 (1965), 286-294.

[20] R. Schoen, Conformal deformation of a Riemannian metric to constant scalar curvature, J. Differential Geom. 20 (1984), no. 2, 479-495.

[21] G. Simonett, Center manifolds for quasilinear reaction-diffusion systems, Differential Integral Equations 8 (1995), no. 4, 753-796.

[22] H. Triebel, Interpolation Theory, Function Spaces, Differential Operators, Second edition. Johann Ambrosius Barth, Heidelberg, 1995.

[23] K. Yano, Differential Geometry on Complex and Almost Complex Spaces, International Series of Monographs in Pure and Applied Mathematics, Vol. 49 A Pergamon Press Book. The Macmillan Co., New York 1965.

[24] R. Ye, Ricci flow, Einstein metrics and space forms, Trans. Amer. Math. Soc. 338 (1993), no. 2, 871-896.

[25] P. B. Zhao and H. Z. Song, Quasi-Einstein hypersurfaces in a hyperbolic space, Chinese Quart. J. Math. 13 (1998), no. 2, 49-52.

[26] P. B. Zhao and X. P. Yang, On quasi-Einstein field equation, Northeast. Math. J. 21 (2005), no. 4, 411-420.

[27] _ On stationary hypersurfaces in Euclidean spaces, Acta Math. Sci. Ser. B Engl. Ed. 26 (2006), no. 2, 349-357.

Peibiao Zhao

Department of Applied Mathematics

Nanjing University of Science and Technology

NANJING 210094, P. R. CHINA

E-mail address: pbzhao@mail.njust.edu.cn

QIHUI CAI

Department of Applied Mathematics

Nanjing University of Science and Technology

NANJing 210094, P. R. ChinA

E-mail address: hqcai2000@sina.com.cn 